\title{
2400. Numerical simulation and experimental research of spectral noise characteristics for the four-wheel landing gear
}

\author{
Ting Li ${ }^{1}$, Jin-hua Zhang ${ }^{2}$, Bao-zeng Wang ${ }^{3}$, Tao Xue ${ }^{4}$ \\ ${ }^{1,4}$ School of Computer Science, Xi' an Polytechnic University, Xi' an 710048, China \\ ${ }^{2,}{ }^{3}$ School of Mechanical Engineering, Xi'an Jiaotong University, Xi'an 710048, China \\ ${ }^{4}$ Corresponding author \\ E-mail: ${ }^{1} 13484462161 @ 163 . c o m,{ }^{2} j j s h u a 1 @ m a i l . x j t u . e d u . c n,{ }^{3} b z \_w a n g @ s t u . x j t u . e d u . c n$, \\ ${ }^{4} x u e t a o @ 163 . c o m$
}

Received 1 May 2016; received in revised form 4 October 2016; accepted 19 October 2016 DOI https://doi.org/10.21595/jve.2016.17126

Check for updates

Abstract. Numerical simulation and wind tunnel experiment were conducted for flow field and flow noise characteristics of four-wheel landing gear. Under the landing speed, large eddy simulation method was applied to simulate the unsteady turbulent flow field around the landing gear. In addition, vortex-acoustic theory was also employed to compute the intensity and position of sound source, and integral method of FW-H equation was used to solve the sound field generated from different components and their combinations, analyze the noise generation mechanism, spectral characteristics and far-field directivity. Meanwhile, the contribution of each component to the total noise was assessed. The aerodynamic acoustic experiment was conducted for landing gear in the wind tunnel, and a microphone was applied to measure and obtain the spectral characteristics of noise, which was compared with the numerical computation result. As shown from the result, a good agreement was found between the experimental and numerical results. Besides, it was shown from the simulation result that small parts ignored in the computational sound field played a greater impact on prediction result of noise, especially high-frequency noise. As shown from landing gear vorticity and sound pressure distribution, sound source was mainly distributed in the solid surface of various components for the landing gear. Additionally, sound source was also occurred in the vortex shedding positions behind each component. Greater energy and density of the sound source were found in the gap formed between tire and bogie and in the pillar stairs. The tire noise showed maximum contribution to the total noise, followed by the bogie noise, while the pillar noise was minimal. The total noise spectrum was closest to the tire noise spectrum.

Keywords: wind tunnel experiment, flow noises, four-wheel landing gear, tire noise spectrum.

\section{Introduction}

The airframe noise in landing stage was mainly from the landing gear and high lift devices $[1,2]$. However, the landing gear noise occupied $25 \%$ of the total aircraft noise when the engine was stopped and the wing was folded [3]. Nevertheless, numerical simulation and experimental research of landing gear noise was difficult due to the complex geometry of landing gear, its strong interaction with surrounding fluids [4-6], as well as wide acoustic band range, extensive spread range and relatively low energy and other factors. The landing gear noise problem has still been one of the difficulties in airframe noise studies so far.

Currently, a lot of researches have been conducted in the aspect of aerodynamic noise regarding the landing gear. Kourbatski [7] presented a computational investigation of the problem of unsteady flow past a rudimentary four-wheel landing gear configuration and associated far-field noise radiation, and numerical values of pressure spectra at selected location on the landing gear were compared with experimental data. Long [8] applied separate vortex method to simulate unsteady turbulent flow field on the basis of front landing gear model of the airplane, extracted the intensity and distribution information of sound source, and solved sound field result by the integral method of FW-H equation. In her conclusion, the greatest impact on far-field radiation 
noise was played by the tire noise of the landing gear, followed by bogie and pillar. The far-field noise produced by a Rudimentary Landing Gear (RLG) was investigated using Delayed Detached Eddy Simulation (DDES) coupled with a Ffowcs-Williams Hawkings (FWH) integral [9]. Computational results are presented for the RLG geometry using three unstructured grids of quasi-nested refinement. DDES has the potential to become a viable tool for evaluating low noise gear designs during the early design phases before wind tunnel and flight testing have commenced. Liu [10] computed the landing-gear noise using high-order finite difference schemes, and both aerodynamic and aero-acoustic results are compared to wind tunnel measurements and are found to be in good agreement. For Dobrzynski [11], a wind tunnel test was conducted for front landing gear and main landing gear of aircraft A320, the noise source positions was obtained on the basis of microphone array, and the low-noise landing gear was designed on the former basis. Spalart [12] computed the far-field and near-field noise of the landing gear numerically, studied the noise spectrum characteristics of landing gear under different yaw angles, and found that yaw angle affected the landing gear noise quite seriously. The mentioned researches on the landing gear are not deep enough, and the analysis of the components was not conducted. In addition, the research was only conducted based on experiment or simulation separately.

Large eddy simulation was applied in the paper to calculate the flow filed numerically. Then, the acoustic field characteristics on the surface of the landing gear were studied on the basis of flow field results and verified through experiments. Finally, the integral method was employed to study the impact of different components of landing gear on the far-field noise.

\section{Theoretical backgrounds}

\subsection{Flow field calculation}

The incompressible viscous flow field could be applied in the external flow field of the landing gear, whose basic equation was as follows:

$$
\begin{aligned}
& \frac{\partial u_{i}}{\partial x_{i}}=0, \\
& \frac{\partial u_{i}}{\partial t}+u_{j} \frac{\partial u_{i}}{\partial x_{j}}=-\frac{1}{\rho} \frac{\partial p}{\partial x_{i}}+\mu \frac{\partial^{2} u_{i}}{\partial x_{j} \partial x_{j}}
\end{aligned}
$$

wherein: $u_{i}$ and $u_{j}$ are flow field speeds around the landing gear, $x_{i}$ and $x_{j}$ are components of the coordinate, $\rho$ represents the air density, $p$ means pressure and $\mu$ expresses the aerodynamic viscosity.

Large eddy simulation equations were still N-S equation of incompressible fluid, and large-scale speed in LES was the filtering speed, which was defined as follows:

$\bar{u}_{i}(x, t)=\int G\left(x, x^{\prime}, \Delta\right) u_{i}(x) d x^{\prime}$,

wherein: $G\left(x, x^{\prime}, \Delta\right)$ represents the filter function, which is applied to calibrate large eddy and filter small eddy. $\Delta$ means the size of the filter grid. It is assumed that the filtration process can be exchanged with the process of derivation, which can be applied in N-S equation of incompressible viscous fluid. Regardless of $G$ form, the following equation can be always obtained:

$$
\begin{aligned}
& \frac{\partial \bar{u}_{i}}{\partial x_{i}}=0, \\
& \frac{\partial \bar{u}_{i}}{\partial t}+\frac{\partial \bar{u}_{i} \bar{u}_{j}}{\partial x_{j}}=-\frac{1}{\rho} \frac{\partial \bar{p}}{\partial x_{i}}+\mu \frac{\partial^{2} \bar{u}_{i}}{\partial x_{j} \partial x_{j}}+\frac{\partial \bar{\tau}_{i j}}{\partial x_{j}},
\end{aligned}
$$


wherein: $\bar{\tau}_{i j}=-\left(\overline{u_{i} u_{j}}-\bar{u}_{i} \bar{u}_{j}\right)$ is SGS Renolds Stress, which is a new unknown quantity that needs modeling.

For making equations closed, it is assumed that SGS stress can be expressed as the following equation in accordance with basic SGS model of Smagorinsky:

$\tau_{i j}-\tau_{k k} \delta_{i j} / 3=-2 \mu_{i} \bar{S}_{i j}$

wherein: $\mu_{i}$ represents the viscosity coefficient of the subgrid turbulence and $\bar{S}_{i j}$ means the strain tensor component under the solving scale. $\bar{S}_{i j}$ is defined as the following equation:

$\bar{S}_{i j}=\frac{1}{2}\left(\frac{\partial \bar{u}_{i}}{\partial x_{j}}+\frac{\partial \bar{u}_{j}}{\partial x_{i}}\right)$.

As LES could only make transient calculation with a higher requirement for the initial flow field, the calculation of steady flow field was firstly solved by the application of standard $k-\varepsilon$ turbulence model, whose equations were shown below:

$\frac{\partial(\rho k)}{\partial t}+\frac{\partial\left(\rho k u_{i}\right)}{\partial x_{i}}=\frac{\partial}{\partial x_{j}}\left[\left(\mu+\frac{\mu_{t}}{\sigma_{k}}\right) \frac{\partial k}{\partial x_{j}}\right]+\mu_{t} \frac{\partial u_{j}}{\partial x_{i}}\left(\frac{\partial u_{j}}{\partial x_{i}}+\frac{\partial u_{i}}{\partial x_{j}}\right)-\rho \varepsilon$,
$\frac{\partial(\rho \varepsilon)}{\partial t}+\frac{\partial\left(\rho \varepsilon u_{i}\right)}{\partial x_{i}}=\frac{\partial}{\partial x_{j}}\left[\left(\mu+\frac{\mu_{t}}{\sigma_{\varepsilon}}\right) \frac{\partial \varepsilon}{\partial x_{j}}\right]+C_{1} \mu_{t} \frac{\varepsilon}{k} \frac{\partial u_{j}}{\partial x_{i}}\left(\frac{\partial u_{j}}{\partial x_{i}}+\frac{\partial u_{i}}{\partial x_{j}}\right)-C_{2} \rho \frac{\varepsilon^{2}}{k}$,

wherein: $k$ represents the turbulent kinetic energy, $\varepsilon$ is the turbulent dissipation rate, $\mu_{t}$ is the turbulent viscosity coefficient, $\mu_{t}=C_{\mu} \rho k^{2} / \varepsilon$, and $C_{\mu}$ is the constant of turbulence. Under normal circumstances, $C_{\mu}=0.09 . C_{1}, C_{2}, \sigma_{k}$, and $\sigma_{\varepsilon}$ are empirical constants. $C_{1}=1.47, C_{2}=1.92$, $\sigma_{k}=1.0$ and $\sigma_{\varepsilon}=1.33$ were extracted for the calculation in the paper.

\subsection{Computation of sound field}

Sound field simulation was based on FW-H equation, as shown in Eq. (10). The sound radiation source was represented by the right three terms in FW-H equation, wherein the first term represented the turbulent stress of the fluid itself, with quadrupole characteristics; the second term expressed the divergence of unsteady force exerted on certain interfaces, with dipole characteristics; and the third term was the unsteady mass flow entering into the fluid, with monopole characteristics:

$$
\begin{aligned}
& \frac{1}{a_{0}^{2}} \frac{\partial^{2} p^{\prime}}{\partial t^{2}}-\nabla^{2} p^{\prime}=\frac{\partial^{2}}{\partial x_{i} \partial x_{j}}\left\{T_{i j} H(f)\right\}-\frac{\partial}{\partial x_{i}}\left\{\left[P_{i j} n_{j}+\rho u_{i}\left(u_{n}-v_{n}\right)\right] \delta(f)\right\} \\
& \quad+\frac{\partial}{\partial t}\left\{\left[\rho_{0} v_{n}+\rho\left(u_{n}-v_{n}\right)\right] \delta(f)\right\},
\end{aligned}
$$

wherein: $a_{0}$ is the speed of sound, $p^{\prime}$ is the fluctuating sound pressure, $T_{i j}$ is the stress tensor of Lighthill, and $P_{i j}$ is a stress tensor of the compressible fluid. $u_{i}$ is the velocity component of fluid in the direction of $x_{i}, u_{n}$ is the normal velocity of fluid on the surface of $f=0 . v_{n}$ is the normal component of velocity on the object surface. $H(f)$ expresses generalized function of Heaviside and $\delta(f)$ is nowhere continuous function. Without any volume integral, all sound sources within the integral plates can be calculated by FW-H equation. The part surface was defined as the integral plate in the simulation herein. 


\section{Computation of noise on landing gear surface}

Great difficulties for geometry modeling, mesh generation and aerodynamic noise computation would be caused by the details in the landing gear. Therefore, the details of the simulation model needed to be simplified under the premise of the remained main features of the sound field, as shown in Fig. 1. Such simplification retained the most structures of the real landing gear and only leaved out small components, but it would not have a significant influence on the calculation result. At present, the reported models of the landing gear were basically shown in Fig. 1. They could be considered as standard models. For instance, the model of the landing gear based on simplification in literature [7-13] numerically computed the aerodynamic noise of the landing gear and obtained some meaningful conclusions. The tire diameter of the landing gear was D. The axial distance of front and rear tires was $1.16 \mathrm{D}$, pillar width and length were both $0.25 \mathrm{D}$, distance between tires was $0.88 \mathrm{D}$, bogie height and width were both $0.3 \mathrm{D}$, and tire width was $0.37 \mathrm{D}$. On the basis of the sizes, the established three-dimensional model of the landing gear was shown in Fig. 2. The sizes of four wheels of the landing gear were remained consistent and symmetrical with respect to the bogie.
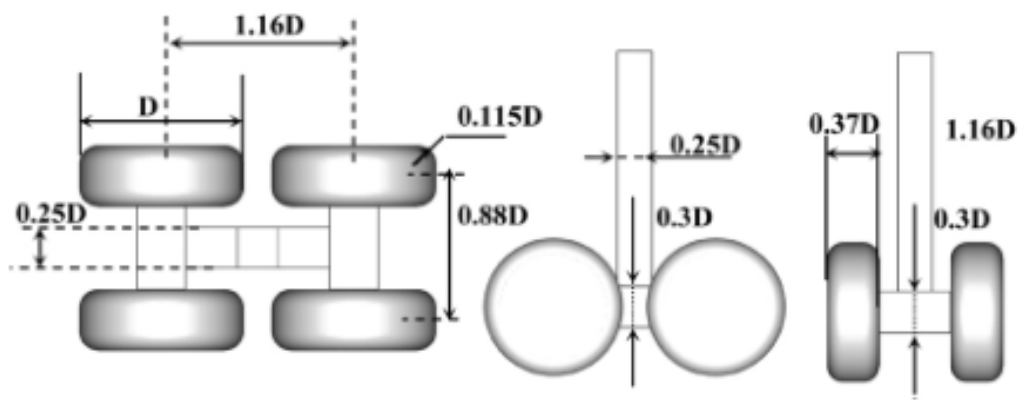

Fig. 1. Sizes of main parts of landing gear
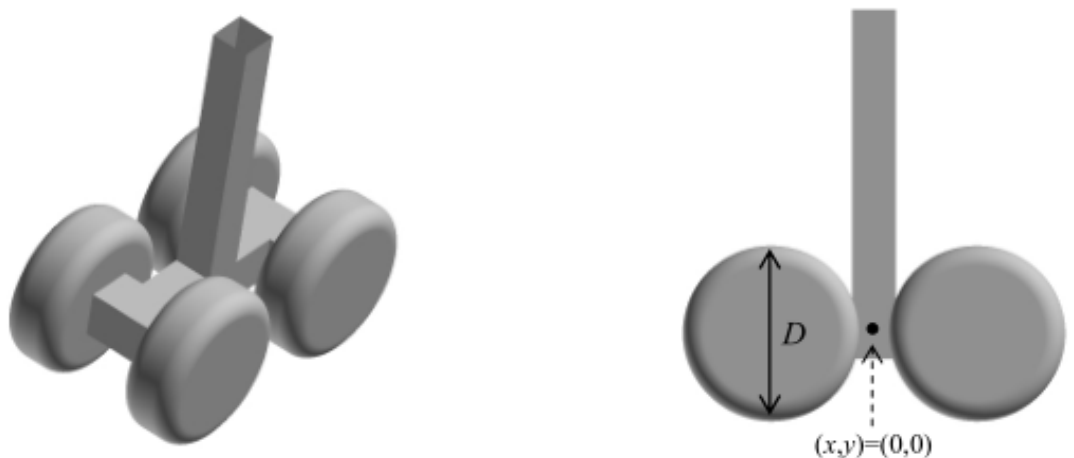

Fig. 2. Geometric model of landing gear

In the calculation process of flow field for the landing gear, the calculation domain needed to be divided firstly. The pillar axis was 3.5D away from the entrance and 6.5D away from the exit. The incoming flow surface was defined as the speed inlet, the entrance plane was defined as the free outlet, and other surfaces and model surfaces were all defined as the wall. The established three-dimensional solid model was converted to the common output format, and then imported into the mesh generation software GRIDGEN. According to the database of the geometric model, the structured grid was created as shown in Fig. 3(a). A total of 1,458,961 hexahedral elements were included in the computational domain. As shown in Fig. 2, the tire and bogie of the landing gear were rigidly connected. 4 tires were connected with both sides of the bogie. In the process of generating meshes, mesh generation was firstly conducted for the tire. Mesh size was $2 \mathrm{~mm}$. Then, 
mesh generation was conducted for the bogie. Mesh size was also $2 \mathrm{~mm}$. Finally, the tire and bogie were rigidly connected to form the model as shown in Fig. 3(b).

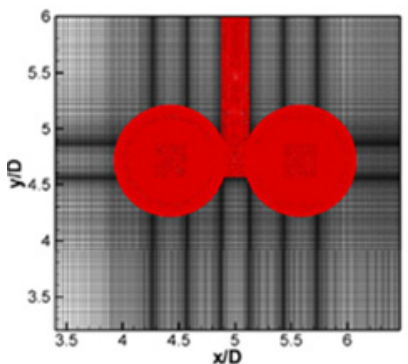

a) Computational domain of landing gear



b) Local elements

Fig. 3. Computational domain and local grid of landing gear

Based on the aerodynamic characteristic of the landing gear, the SPL contours can be obtained. SPL contours of the landing gear at $600 \mathrm{~Hz}$ and $800 \mathrm{~Hz}$ were extracted as shown in Fig. 4, which showed that the sound pressure at $600 \mathrm{~Hz}$ was significantly greater than that at $800 \mathrm{~Hz}$.

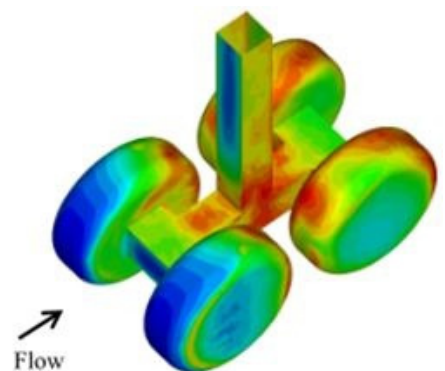

a) $600 \mathrm{~Hz}$

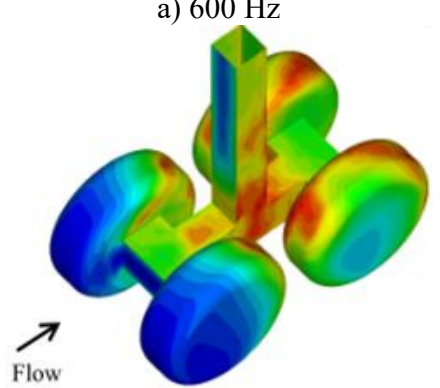

c) $800 \mathrm{~Hz}$

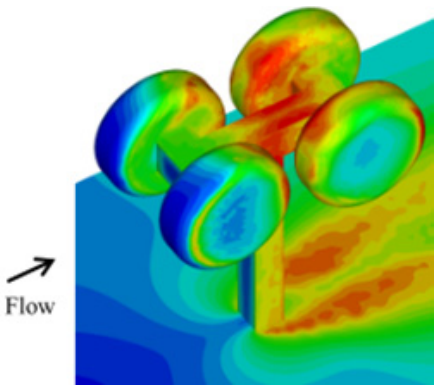

b) $600 \mathrm{~Hz}$

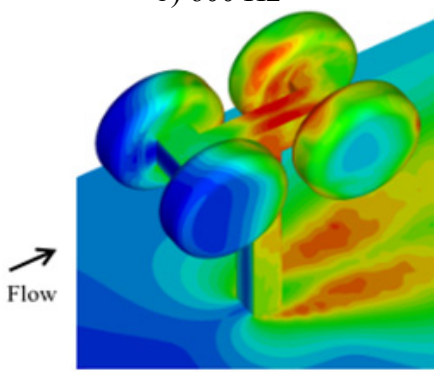

d) $800 \mathrm{~Hz}$

Fig. 4. SPL contours of landing gear

\section{Experimental verification for numerical calculation results}

Wind tunnel test was conducted for the landing gear to verify the accuracy concerning numerical simulation of the landing gear noise, as shown in Fig. 5. The landing gear was fixed in the wind tunnel with the certain amount of incoming flow applied oppositely. The full-size model of the landing gear in an airplane was used as the test sample, which was mounted on the wind tunnel ground. Microphone was placed near the landing gear to obtain the sound pressure signals, and the experimental time was $2 \mathrm{~s}$, and each test was conducted for three times. Finally, the average value was the final result. The experimental process was shown in Fig. 6. When the signal was obtained, it was imported into the computer. Then, the signal was processed by pulse software to the sound pressure level. 


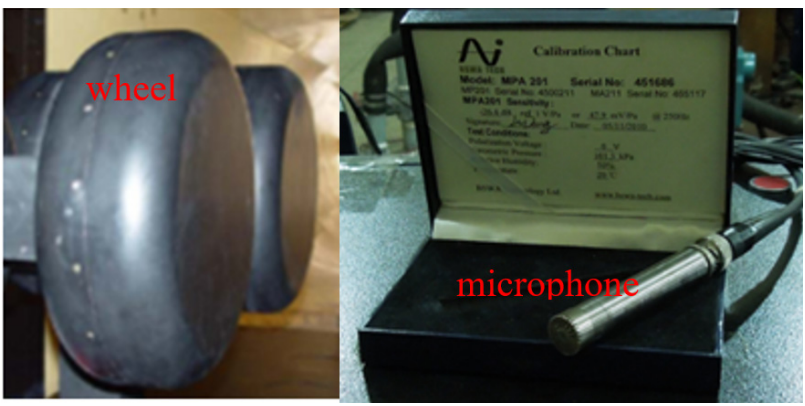

Fig. 5. Wind tunnel tests of landing gear

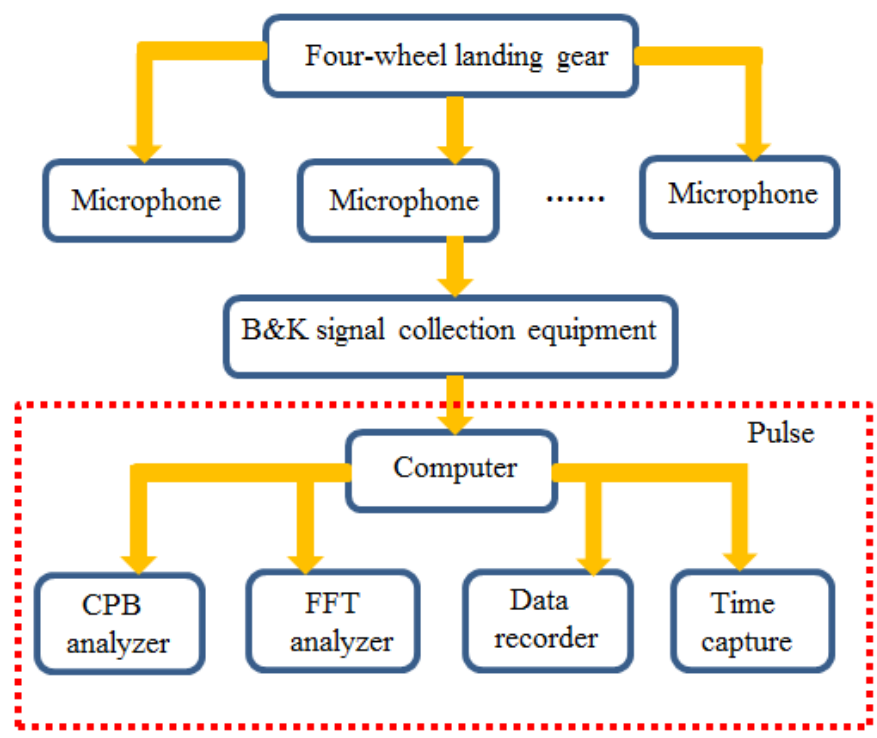

Fig. 6. The experimental process

The test result was compared with the numerical simulation result, as shown in Fig. 7. It was indicated the basically consistent overall trend between the simulation and experimental results, as well as certain differences existing in terms of values.

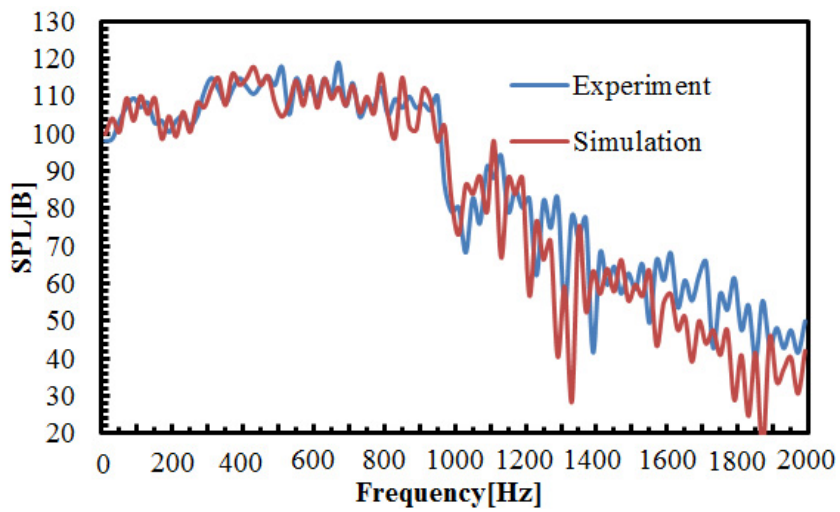

Fig. 7. SPL comparison between simulation and experiment

The simulation model produced its sound through the interaction of the uniform incoming flow and airflow. However, since the wind-ward area of the model had the ratio of $23 \%$ against the 
opening test of the wind tunnel in the experiment, the experimental flow field was of greater difference with the uniform incoming flow, and the experimental test conditions was considerably different from the actual landing condition, which was therefore an important source of errors existing in the simulation and experimental measurement results. Some model details were ignored in the geometrical modeling of the simulation model, but they had mainly generated high-frequency sound waves, thus causing errors in the high frequency band. Generally speaking, the landing gear noise calculated through such model was reliable.

\section{Analysis of noise characteristics for landing gear components}

Solid surfaces of bogie, tires, pillar and the whole landing gear were selected as the integral surface, as shown in Fig. 8. The noise generated by components and the entire landing gear model was calculated in the far field. The position of $R_{1}$ and $R_{2}$ was as shown in Fig. 9. They were 10D away from the tire on the right-hand side horizontally and faced the center of front and back tires directly. The measurement points $R_{1}$ and $R_{2}$ in the far field were taken as the example to illustrate the noise characteristics of various components as well as the contribution of various components to the total noise. The spectral simulation curves of each component at two points were shown in Fig. 10 .

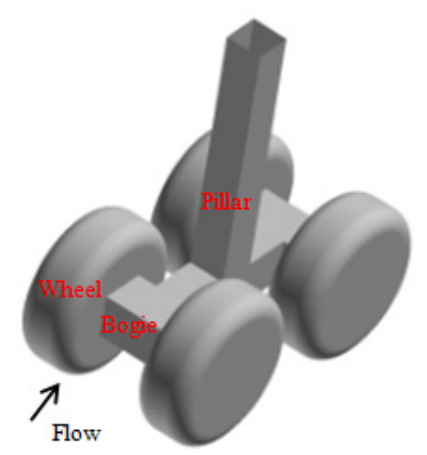

Fig. 8. Integral surface of various components for landing gear
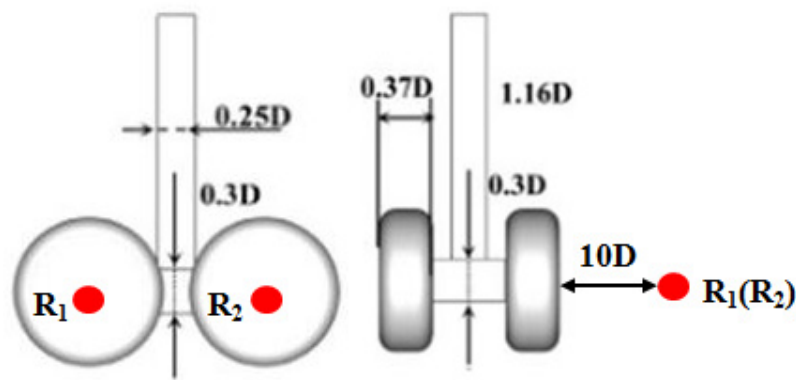

Fig. 9. Position of the observation point $R_{1}$ and $R_{2}$

In the lateral position $R_{1}$, the narrow-band peak was appeared in the spectral curve of bogie at about $696 \mathrm{~Hz}$, with the peak change of $20 \mathrm{~dB}$. In the position $R_{2}$, the characteristics of broadband noise were presented in the spectrum of bogie, without apparent narrow-band peak value. As shown from the simulation result, the sound source with the frequency of about $696 \mathrm{~Hz}$ was included in the bogie noise, including certain directivity. As shown from the sound source localization, strong sound source was presented in the gap between bogie and tire, indicating that the sound source in the bogie was generated from the cavity formed by the fluid passing through the gap between bogie and tire, which could also be seen in Fig. 4. Due to the reflection of tire surface on the cavity noise, the sound waves generated from the sound source was mainly spread towards both sides of the tire. Therefore, in the lateral position, strong energy and apparent peak of noise can be recognized in the narrow-band peak; while in the position right behind landing gear, small energy and unapparent peak of noise were found in the narrow-band peak. When the analyzed frequency changed from $1100 \mathrm{~Hz}$ to $1800 \mathrm{~Hz}$, SPL curve of various components presented a large fluctuation. It was mainly because the analyzed frequency band was high frequency for the landing gear. The modal density of the landing gear at the high frequency band was relatively higher. Various components had serious resonance under the action of fluid, which was manifested as a large fluctuation in SPL curve. 


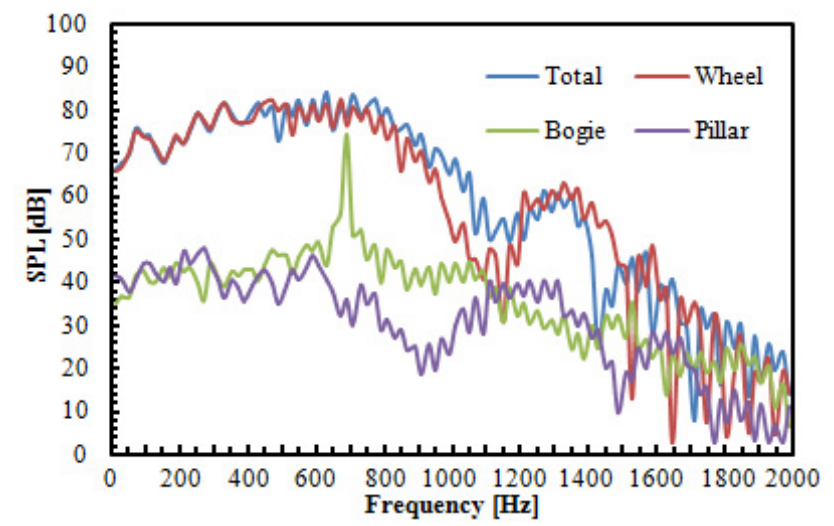

a) SPL comparison regarding different integral surfaces in point $R_{1}$

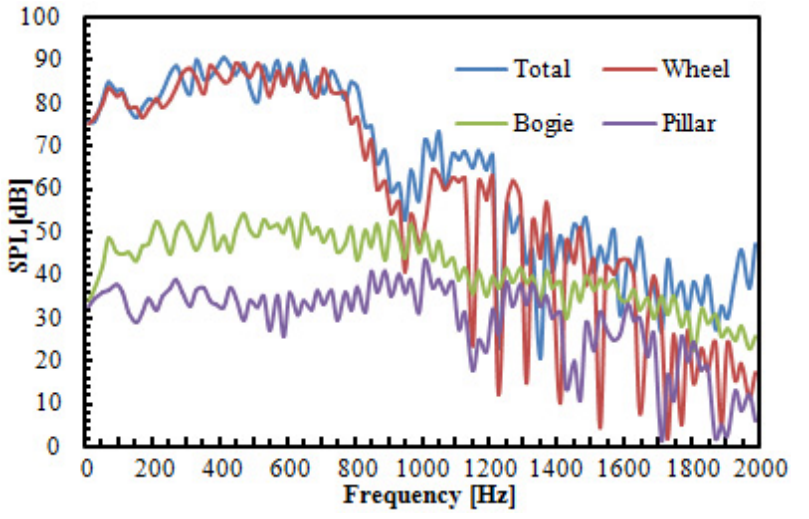

b) SPL comparison regarding different integral surfaces of point $R_{2}$

Fig. 10. SPL comparison regarding different integral surfaces in points $R_{1}$ and $R_{2}$

As shown from Fig. 10, the total noise frequency was closest to tire noise frequency of the model. Firstly, SPL of each component was transformed into sound pressure. Secondly, the average sound pressure of various components at observation points was obtained and average SPL was further computed. The average noise of various components generated at two points was listed in Table 1. It was seen that the tire noise had the maximum contribution to the aerodynamic noise of the entire model, followed by bogie noise contribution, but pillar noise made lowest contribution to the noise of the entire model.

Table 1. Average SPL of various components at points $R_{1}$ and $R_{2}$

\begin{tabular}{|c|c|c|c|c|}
\hline Point & Pillar & Bogie & Wheel & Total \\
\hline$R_{1}$ & 35.61 & 38.23 & 73.33 & 74.42 \\
\hline$R_{2}$ & 32.35 & 40.44 & 72.19 & 73.21 \\
\hline
\end{tabular}

\section{Conclusions}

The near-field and far-field noises of the landing gear were studied based on wind tunnel test and numerical simulation. Small components of simulation model had a significant influence on the prediction result of high-frequency noises. However, the computational result of the numerical model was basically the same with the experimental result. The flow noise of the landing gear could be effectively computed. In addition, it could be seen from sound pressure distribution of the landing gear that strong sound source was mainly distributed in the solid surface of various components of the landing gear. A lot of sound source was also in the position of vortex shedding at the back of various components. The energy and density of sound source were relatively larger 
at the position of a gap formed by the tire and wheel fork and on the steps of the bogie. Finally, an analysis was conducted on the contribution of various components of the landing gear. Results found that the tire noise of the landing gear made the great contribution to the overall noise, followed by the noise of wheel fork. Bogie noises made the smallest contribution to the overall noise. The frequency spectrum of the overall noise was the closest to that of tire noises. Therefore, the subsequent studies will take optimization measures to reduce the aerodynamic radiation noise, including adopting sound package, new materials or the redesign of structures. The actual structure of the landing gear could be redesigned through these studies to effectively improve the radiation noise brought by the landing gear when airplanes were in the process of landing and take-off, which had a great application value to aeronautical engineering.

\section{References}

[1] Qiao W. Y., Xu K. F., Wu Z. W. Noise radiation of large-scale commercial aircraft in take-off and landing. Acta Aeronautica et Astronautica Sinica, Vol. 29, Issue 3, 2008, p. 534-541.

[2] Dobrzynski W. Almost 40 years of airframe noise research: what did we achieve. Journal of Aircraft, Vol. 47, Issue 2, 2010, p. 353-367.

[3] Monclar P. Technology programs for landing gear systems. AIAA/CEAS International Air and Space Symposium and Exposition, 2003, p. 1-9.

[4] Boorsma K., Zhang X., Molin N. Landing gear noise control using perforated fairings. Acta Mechanical Sinica, Vol. 26, Issue 1, 2010, p. 159-174.

[5] Nie H., Wei X. H. Key technologies for landing gear of large civil aircrafts. Journal of Nanjing University of Aeronautics and Astronautics, Vol. 40, Issue 4, 2008, p. 427-432.

[6] Lazos B. S. Mean flow features around the inline wheels of four-wheel landing gear. AIAA Journal, Vol. 40, Issue 2, 2002, p. 193-198.

[7] Kourbatski K. A., Viti V., Menter F., et al. Rudimentary landing gear noise predictions using scaleresolving simulations: Part 1 - Incompressible approach. AIAA Paper, Vol. 463, 2013.

[8] Long S. L., Nie H., Xue C. J. Simulation and experiment on aero-acoustic noise characteristics of aircraft landing gear. Acta Aeronautica et Astronautica Sinica, Vol. 33, Issue 6, 2012, p. 1002-1013.

[9] Langtry R. B., Larssen J. V., Winkler C. M., et al. DDES and acoustic prediction of rudimentary landing gear experiment using unstructured finite volume methods. Flow, Turbulence and Combustion, Vol. 91, Issue 3, 2013, p. 717-745.

[10] Liu W., Kim J. W., Zhang X., et al. Landing-gear noise prediction using high-order finite difference schemes. Journal of Sound and Vibration, Vol. 332, Issue 14, 2013, p. 3517-3534.

[11] Dobrzynski W., Roland E., Pott-Pollenske M. Research at DLR towards airframe noise prediction and reduction. Aerospace Science and Technology, Vol. 12, Issue 1, 2008, p. 80-90.

[12] Spalart P. R., Shur M. L., Strelets M. K., et al. Initial noise predictions for rudimentary landing gear. Journal of Sound and Vibration, Vol. 330, Issue 17, 2011, p. 4180-4195.

[13] Spalart P. R., Shur M. L., Strelets M. K., et al. Reprint of: towards noise prediction for rudimentary landing gear. Procedia IUTAM, Vol. 1, 2010, p. 283-292.

[14] Li Y., Smith M., Zhang X. Measurement and control of aircraft landing gear broadband noise. Aerospace Science and Technology, Vol. 23, Issue 1, 2012, p. 213-223.



Ting Li received the M.S. degree in artificial intelligence and knowledge engineering from Xi'an University of Technology, Xi'an, China, in 2009. Her current research interests include brain-machine interface and robotics. She is currently working toward the Ph.D. degree in the School of Mechanical Engineering, Xi'an Jiaotong University, Shaanxi Province, China, and a visiting scholar in the School of Mechanical Engineering, University of California, Berkeley. 


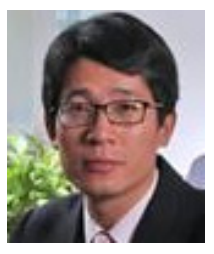

Jinhua Zhang received the B.Sc. degree in mechanical engineering and automation from School of Mechanical Engineering, Xi'an Jiaotong University, Shaanxi Province, China, in 2000, the M.Sc. degree in mechanical design theory from School of Mechanical Engineering, Xi'an Jiaotong University, Shaanxi Province, China, in 2003, and the Ph.D. degree in instrumentation science and technology from School of Mechanical Engineering, Xi' an Jiaotong University, Shaanxi Province, China, in 2008. He is currently an Assistant Professor in the School of Mechanical Engineering, Xi'an Jiaotong University. His research interests are in bio-electricity signals based human computer interaction and biological robot technology. He is a member of the IEEE.

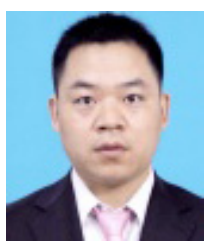

Baozeng Wang received the M.S. degree in Mechanical and Electrical Engineering from Lanzhou University of Technology, Gansu, China, in 2010. He current research interests include brain-machine interface, EEG decoding, and robotics. He is currently working toward the Ph.D. degree in the School of Mechanical Engineering, Xi'an Jiaotong University, Shaanxi Province, China.

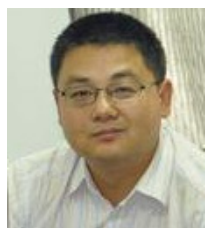

Tao Xue was born in Shaanxi Province, China, in 1973. He received his B.S. and M.S. degrees from Xi'an Jiaotong University of China (XJTU) and Northwest University of China (NWU) in 1995 and 2000, respectively, and received the Ph.D. degree in computer software and theory from the XJTU in 2005. He is currently an associate professor in College of Computer Science at Xi'an Polytechnic University. His research interests include cloud computing, big data and content-based networking. 